its current form is already a major breakthrough for on-chip optical modulation techniques. It outperforms current devices in several aspects, and most importantly, it offers the possibility of integration with other nanophotonic structures on a single monolithic chip. Further developments in this technology may prove to be game-changing within photonics research.
Michał Karpiński

Faculty of Physics, University of Warsaw, Warsaw, Poland.

e-mail:mkarp@fuw.edu.pl

Published online: 24 April 2019

https://doi.org/10.1038/s41566-019-0418-2

References

$\square \quad$ 1. Sounas, D. L. \& Alù, A. Nat. Photon. 11, 774-783 (2017).
2. Torres-Company, V. \& Weiner, A. M. Laser Photon. Rev. 8 , 368-393 (2014)

3. Kues, M. et al. Nat. Photon. 13, 170-179 (2019)

4. Karpiński, M., Jachura, M., Wright, L. J. \& Smith, B. J. Nat. Photon. 11, 53-57 (2017)

5. Midolo, L., Schliesser, A. \& Fiore, A. Nat. Nanotechnol. 13,11-18 (2018).

6. Fan, L., Zou, C.-L., Zhu, N. \& Tang, H. X. Nat. Photon. https://doi.

org/10.1038/s41566-019-0375-9 (2019)..

7. Fan, L. et al. Nat. Photon. 10, 766-770 (2016).

8. Aspelmeyer, M., Kippenberg, T. J. \& Marquardt, F. Rev. Mod. Phys. 86, 1391-1452 (2014).

9. Wang, C., Zhang, M., Stern, B., Lipson, M. \& Lončar, M. Opt. Express 26, 1547-1555 (2018).

10. Salem, R., Foster, M. A. \& Gaeta, A. L. Adv. Opt. Photon. 5, 274-317 (2013).

\title{
BIOPHOTONICS
}

Corrected: Publisher Correction

\section{Plasmon-enabled disinfection}

The implantation of a synthetic mesh is a standard and common procedure for a patient with a hernia. However, it brings the risk of post-surgical infection caused by bacterial colonization on the surgical mesh surface (see image) that can be difficult to treat. To solve these issues, Ignacio de Miguel and co-workers from Spain have now investigated the use of a light-induced disinfection scheme that exploits the heating of plasmonic gold nanoparticles (GNPs) (Nano Lett. https://doi.org/10.1021/acs. nanolett.9b00187; 2019).

The idea is that the surface of the surgical mesh is chemically modified to anchor GNPs tuned to be resonant at the near-infrared wavelength of $800 \mathrm{~nm}$, inside the first transparency window of biological tissues (650-1,100 nm). On illumination with a laser with an emission wavelength that matches the main absorption peak of the GNRs,

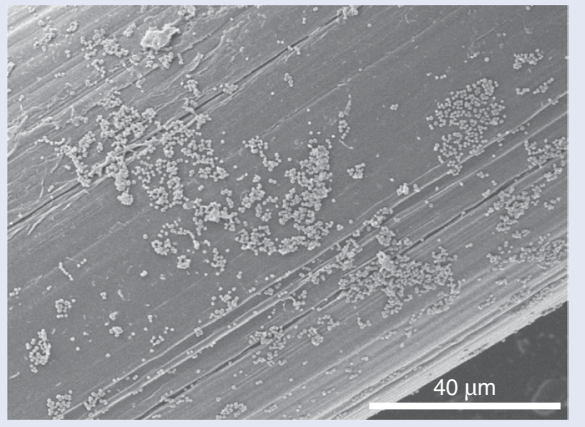

Credit: American Chemical Society

light-induced heating occurs and the local temperature change damages the biofilm produced by bacteria. Unlike antibiotics, because this damage is caused by a physical means, the bacteria cannot adapt to the treatment and become resistant. Furthermore, the near-infrared irradiation can be performed repeatedly after mesh implantation as many times as necessary.

A proof-of-principle experiment was performed with GNP-anchored meshes deliberately infected with Staphylococcus aureus bacteria. The Spanish scientists then systematically changed the irradiation conditions (energy fluence and the pulse duration) and evaluated the impact on the number of bacteria. When the mesh was illuminated with a fluence of $15 \mathrm{~J} \mathrm{~cm}^{-2}$ and pulse duration of $300 \mathrm{~ms}$, the number of bacteria decreased by $97.6 \%$.

The scientists believe that the main mechanism behind the elimination of the biofilm was its loss of adhesion from the mesh, most likely resulting from the denaturation of adhesive exopolysaccharides.

Noriaki Horiuchi

Published online: 24 April 2019

https://doi.org/10.1038/s41566-019-0429-Z

\section{OPTICAL PHYSICS}

\section{En-gauging the light}

Synthetic gauge fields that enable controllable confinement and guiding via bound states in the continuum are demonstrated, offering new ways to confine and manipulate localization of radiation in space and opportunities to new applications of artificial gauge fields in photonics.

\section{Alexander B. Khanikaev}

R ecent developments in engineering artificial photonic media, from photonic crystals to metamaterials and metasurfaces, have demonstrated nearly limitless possibilities in manipulating optical waves. These developments have culminated in topological photonics - a subfield of photonics where control of optical waves is brought to the extreme by completely redefining familiar behaviours. Indeed, a variety of fascinating properties attained by light in topological photonic structures has been demonstrated in the past few years, from reflectionless guiding around defects and obstacles ${ }^{1-4}$, to topological lasing ${ }^{5,6}$, the generation of quantum states of light ${ }^{7,8}$, and the emulation of relativistic phenomena and of higher-dimensional physics exploiting 